\title{
First evidence of Besnoitia bennetti infection (Protozoa: Apicomplexa) in donkeys (Equus asinus) in Belgium
}

Emmanuel Liénard ${ }^{1,2}$, Adriana Nabuco ${ }^{3}$, Sophie Vandenabeele ${ }^{4}$, Bertrand Losson ${ }^{5}$, Irène Tosi ${ }^{5}$, Émilie Bouhsira ${ }^{1,2}$, Françoise Prévot ${ }^{1,2}$, Shukri Sharif ${ }^{1,2}$, Michel Franc ${ }^{1}$, Caroline Vanvinckenroye ${ }^{5}$ and Yannick Caron $^{5^{*}}$

\begin{abstract}
Background: Besnoitiosis is caused by different species of intracellular protozoan parasites belonging to the family Sarcocystidae and affecting multiple host species worldwide. Including B. besnoiti, ten species are described infecting animals. Among ungulates, Besnoitia bennetti infects horses, donkeys and zebras and was described in Africa and in the USA where donkey besnoitiosis is considered as an emerging disease.

Case presentation: A two-year-old male donkey was purchased in May 2016 in poor body condition (cachexia, alopetic areas and pruritus mainly on neck and head) by the present owner in Le Roeulx (Belgium) from a milk producing donkey farm in Frasnes-lez-Buissenal (Belgium). Shortly after its purchase and shearing, the donkey presented with crusts, hyperkeratosis (both flanks and neck) anorexia and cachexia. A treatment with phoxim was given with no improvement. A cutaneous biopsy of hyperkeratotic skin was performed in July. It showed a perivascular eosinophilic infiltrate with a large thick walled cyst located in the dermis containing numerous bradyzoites. This was highly suggestive of besnoitiosis. Several skin biopsy samples were obtained for qPCR analysis and confirmed the presence of Besnoitia spp. DNA. Further laboratory diagnosis tests were performed (western blot and rDNA sequencing) confirming Besnoitia bennetti aetiology for the male. For the female, the punch-biopsy, haematology and GPCR were negatives but the western blot showed the presence of antibodies directed to Besnoitia spp. Further clinical examination performed in August highlighted scleral pinhead sized cysts (pearl) in the right eye and between nares. Another ten-year-old female donkey purchased in France and sharing the same accommodation showed a good clinical condition, but a thorough clinical examination showed the presence of numerous cysts on the inner face of upper labial mucosa. A daily treatment based on sulfamethaxzole and trimethoprim (Emdotrim 60\% Mix ${ }^{\circledast}, 30 \mathrm{mg} / \mathrm{kg}$ ) was given orally and some improvement was noticed.

Conclusion: This is the first evidence of Besnoitia bennetti infection (Protozoa: Apicomplexa) in donkeys (Equus asinus) in Belgium.
\end{abstract}

Keywords: Scleral and labial cysts, Besnoitia bennetti, Donkey, Besnoitiosis, Europe

\section{Background}

Besnoitiosis is caused by different species of intracellular protozoan parasites belonging to the family Sarcocystidae (phylum Apicomplexa) affecting multiple host species worldwide. The cyst-forming parasite, Besnoitia besnoiti, in cattle has received more attention since cattle besnoitiosis was recognized in 2010 as re-emerging

\footnotetext{
* Correspondence: ycaron@pasteur-kh.org

${ }^{5}$ University of Liège, Faculty of Veterinary Medicine, Liège, Belgium Full list of author information is available at the end of the article
}

in Europe by the European Food Safety Authority (EFSA) [1]. Its propagation from historic endemic areas in Europe (Spain, Portugal and southern France) took place during the last two decades and currently at least eight European countries have reported clinical cases in imported or autochthonous animals: Switzerland, Italy, Germany, Croatia, Greece, Hungary, Belgium and Ireland [2-6]. For instance, a western blot revealed infection in a six-year-old Blonde d'Aquitaine bull which was imported in Belgium in 2012 from the Pyrenees

(c) The Author(s). 2018 Open Access This article is distributed under the terms of the Creative Commons Attribution 4.0 International License (http://creativecommons.org/licenses/by/4.0/), which permits unrestricted use, distribution, and 
Mountain endemic region in southern France [7]. The first stage of infection is the acute systemic phase (tachyzoites are present in the blood) characterized by fever, lymphadenopathy and oedematous swelling, sometimes associated with mortality, abortion and male transient or definitive sterility [8]. The second and chronic stage is the scleroderma phase or stage characterized by the development of numerous tissue cysts filled with bradyzoites in the dermis, vaginal and tracheal mucosa and on the ocular sclera, the latter localization being pathognomonic. These cysts are responsible for skin lesions with hyperkeratosis and alopecia [4].

Including $B$. besnoiti, ten species are described, infecting four and six species of large or small animals, respectively [2]. Among ungulates, Besnoitia bennetti infects horses, donkeys and zebras and was described prior to 1992 in Africa (Sudan and South Africa) [2, 9-12] and in the USA [13-15] where donkey besnoitiosis is considered as an emerging disease [16, 17]. The most striking clinical sign shared with cattle besnoitiosis is the development of multifocal pinpoint parasitic cysts in the skin, between the nares, on the internal and external pinnae, limbs and perineum and along the limbal margin of the sclera. Moreover, as observed in B. besnoiti infection, many infected animals remain clinically healthy [18]. Attempts to identify the definitive host for $B$. bennetti have been unsuccessful, precluding researchers from elucidating the parasite's life-cycle in equine infection [18].

In this report, we describe two cases of besnoitiosis in donkeys, for the first time in Belgium, using a full set of diagnostic tools.

\section{Case presentation}

Consultation purpose

In July 2016, a private veterinarian referred a donkey characterised by poor body condition and chronic skin lesions to the Faculty of Veterinary Medicine of Liège (Belgium).

\section{Clinical history}

A two year old male donkey (Grand Noir du Berry breed), was purchased in May 2016 in poor body condition (weight loss, alopecic areas, pruritus mainly on neck and head, dirty long and matted hair) by the present owner in Le Roeulx in Belgium (50³1'49.48"N, $\left.4^{\circ} 06^{\prime} 56.33^{\prime \prime} \mathrm{E}\right)$. This jack came from a milk producing donkey farm in Frasnes-lez-Buissenal, Belgium $\quad\left(50^{\circ} 40^{\prime} 11.31^{\prime \prime} \mathrm{N}, \quad 3^{\circ}\right.$ 37'11.19"E; Fig. 1). A treatment with phoxim (Sarnacuran ${ }^{\oplus}$ ) was given with no improvement.

\section{Clinical descriptions}

Shortly after its purchase, the animal was shorn revealing crusts and hyperkeratosis (on both flanks and the neck). The animal was anorexic and in poor body condition. A closer clinical examination in August highlighted scleral pinhead sized cysts (pearl) in the right eye and between nares (Fig. 2). The rest of the examination was unremarkable.

Another ten year old female donkey (Grand noir du Berry breed), purchased several years ago in France (Loire region) by the same owner and sharing the same accommodation, was in good clinical condition. However, further clinical examination showed the presence of numerous cysts on the inner face of upper labial mucosa.

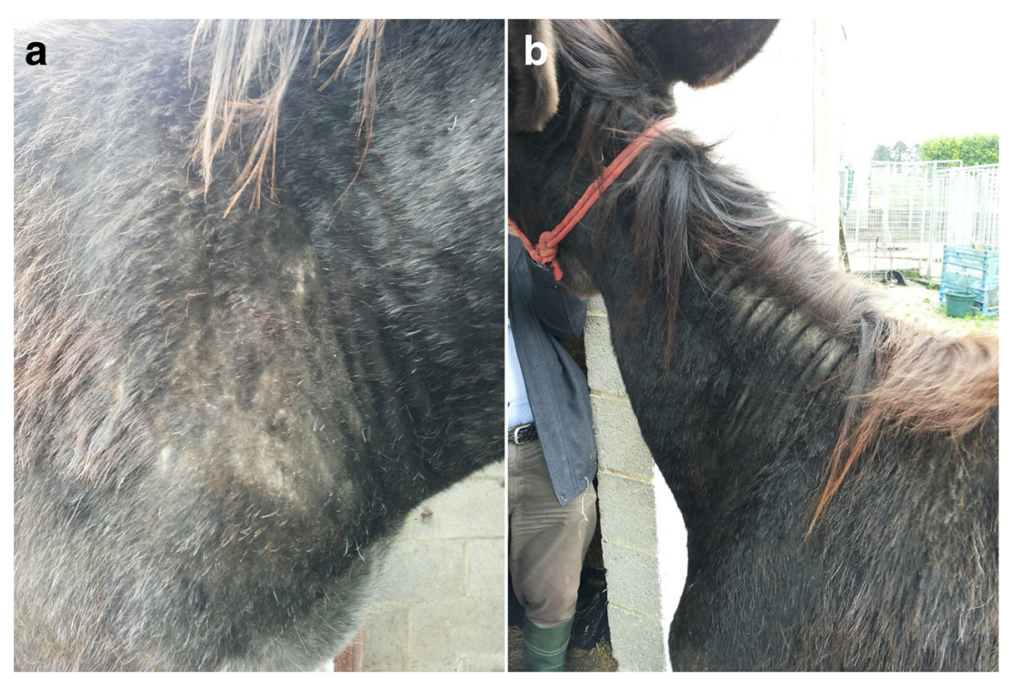

Fig. 1 Patient's skin lesions: a alopetic and crusty area on the right shoulder, b hyperkeratosis (neck) 


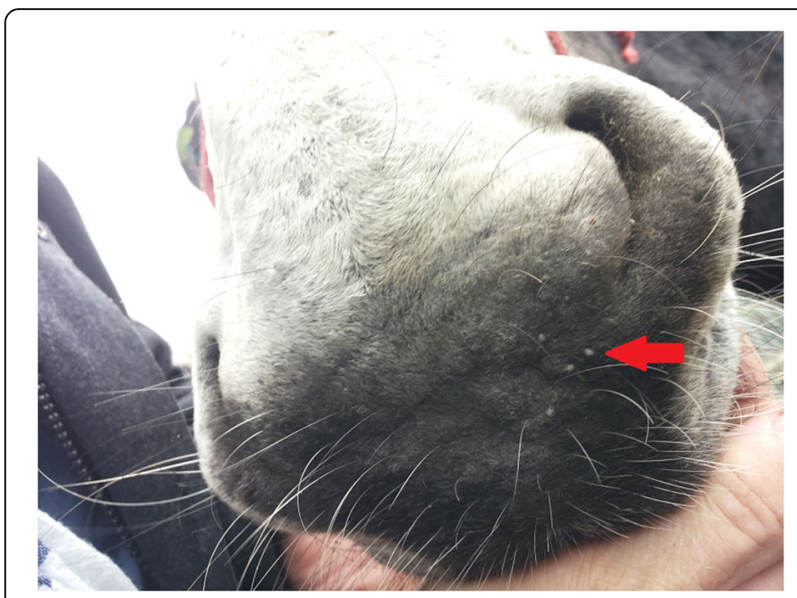

Fig. 2 Scleral pin-head sized cysts (pearl; red arrow) between nares

The two donkeys were kept in a fenced area (below 1 ha). The animals were fed a standard donkey food regimen composed of hay, supplemented with protein-containing grain (oats) and occasional fruits and vegetables. Previous medical treatments included routine vaccinations and prophylactic deworming (based on ivermectin). Other animal species (cats, dogs, chickens and rodents) had free access to the farm and the paddock buildings. Numerous flies were observed in the paddocks (Stomoxys calcitrans and Musca spp.).

\section{Laboratory examination, diagnosis and prognosis Skin scraping}

Skin scrapings were performed in different places (mainly flanks and neck where the lesions were the most obvious) on both animals. A culture on Sabouraud dextrose agar-chloramphenicol $(0.5$ per $1000 \mathrm{~m} / \mathrm{w}$ for three weeks at $37^{\circ} \mathrm{C}$ ) yielded a negative result for ringworm. Microscopic examination of the hair revealed neither fungal spores nor ectoparasites. A Giemsa staining of an impression smear also gave negative results.

\section{Histopathology}

Several skin biopsies were taken using an $8 \mathrm{~mm}$ biopsy punch. Histopathology and DNA extraction for qPCR and PCR were performed on those skin samples. After collection of the sample, the material was stored in $10 \%$ phosphate-buffered formalin for histological examination and in $70 \%$ ethanol for molecular analyses. The formalin-fixed sample was bisected and embedded in paraffin wax at $56{ }^{\circ} \mathrm{C}$, sectioned at $4 \mu \mathrm{m}$, cut and stained with haematoxylin and eosin for routine evaluation. This technique was performed by a veterinary diagnostic laboratory (Medvet, Antwerpen, Belgium).

In the clinically affected patient, focal spongiosis epidermis was observed. In the dermis, there was a perivascular eosinophilic infiltrate and in the deep dermis a large thick-walled $(28 \mu \mathrm{m})$ cyst $(313 \times 344 \mu \mathrm{m})$ packed with bradyzoites present (Fig. 3). With the exception of a perivascular eosinophilic infiltrate, the histopathological preparation from the other animal was unremarkable.

\section{Blood sampling and analysis}

Several blood samples were taken for haematology and biochemistry analysis from both patients. A blood sample from the male gave unremarkable results except a light anaemia [haemoglobin $10.1 \mathrm{~d} / 1$ (normal range 11$19 \mathrm{~d} / \mathrm{l}$ ), red blood cells $5.2210^{6} / \mathrm{mm}^{3}$ (normal range $6.5-12.510^{6} / \mathrm{mm}^{3}$ ), haematocrit $30 \%$ (normal range $32-$ $52 \%$ )], an eosinophilia [15.5\%; $1631 / \mathrm{mm}^{3}$ (normal range $<5 \%$ and $<500 / \mathrm{mm}^{3}$, respectively)] and an increased gamma globulin fraction $[32.8 \% ; 23.9 \mathrm{~g} / \mathrm{l}$ (normal range $13-21 \%$ and 5.5-19 g/l, respectively)]. A blood sample from the female revealed only an eosinophilia (12.7\%, $916 / \mathrm{mm}^{3}$ ).

An in-house western blot was performed to detect specific antibodies in both donkeys. The procedures were adapted from previous publications [19]. Sera were tested from two sampling dates (4th August 2016 and 9th September 2016) at 1:50 dilution for each donkey. Peroxidase-labelled goat anti-horse IgG conjugate [anti-horse IgG (Whole molecule)-peroxidase, Sigma-Aldricht, Saint-Quentin Fallavier, France] was used at 1:150 dilution. A molecular weight marker was used (Precision Plus Protein standards, Bio-Rad, Basel, Switzerland). Serum from a cow chronically infected with $B$. besnoiti was used as a positive control, whereas serum samples from uninfected cattle and donkeys were used as negative controls. Serum was considered positive when at least four out of ten bands of specific tachyzoites antigens $(45,40,37,34$, $30,27,22,17,16$ and $15 \mathrm{kDa}$ ) were observed [5, 16]. Figure 4 shows the results of the western blot. Serum from

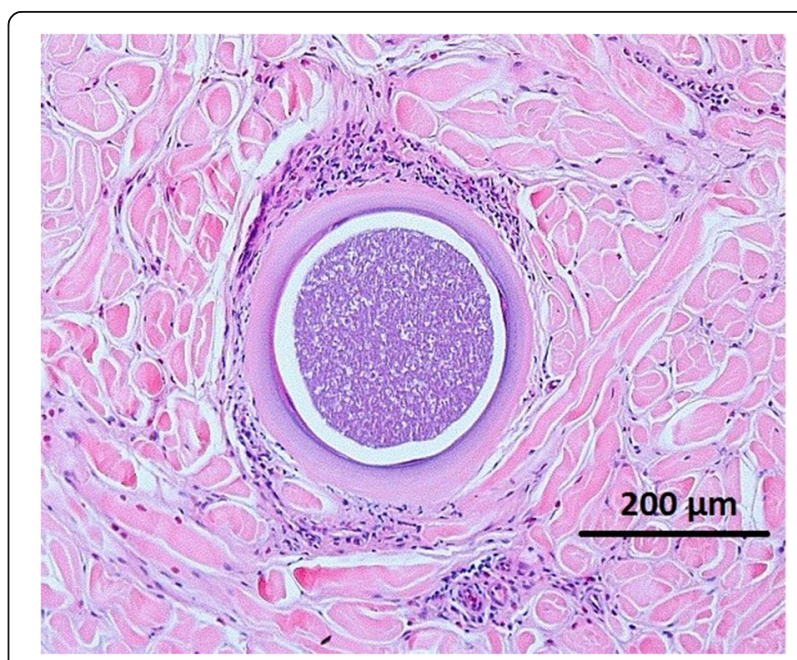

Fig. 3 Histopathological preparation from the male donkey showing a cyst full of bradyzoites (haematoxylin eosin; 10x) 


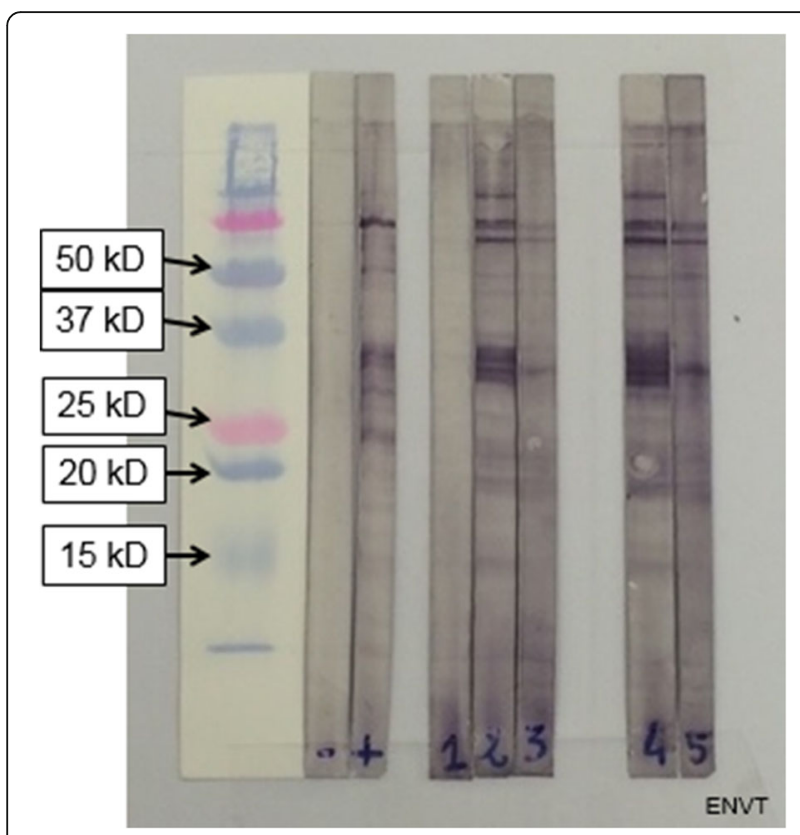

Fig. 4 Western blot results. Lane -: sera from uninfected cattle; Lane +: sera from infected cattle; Lane 1: sera from uninfected donkey; Lane 2: male serum (4/8/2016); Lane 3: female serum (4/8/2016); Lane 4: male serum (9/9/2016); Lane 5: female serum (9/9/2016)

the male donkey exhibited a strong positive result according to criteria defined by others [20] and was even stronger one month later; the female donkey yielded a similar observation.

\section{qPCR}

DNA from skin biopsies $(50 \mathrm{mg}$ ) from both donkeys were extracted with the QIAmp ${ }^{\circ}$ DNA Mini Kit (Qiagen, Courtaboeuf, France) according to the manufacturer's recommendations. Besnoitia spp. internal transcribed spacer 1 (ITS-1) amplification was performed with the commercial PCR kit AdiaVet ${ }^{\mathrm{TM}}$ Besnoitia (AES Chemunex, Bruz, France). The quantitative PCR was performed with the Stratagene MX3005P thermal cycler (Agilent Technologies, La Jolla, CA, USA), and results were analysed using the MxPro QPCR version 4.10 software (Agilent Technologies). A threshold cycle (Ct) value equal to or greater than 40 corresponded to a negative result [20]. Sterile water and genomic DNA from healthy donkeys were used as negative controls. A positive control was provided by a genomic DNA extract of purified $B$. besnoiti tachyzoites cultivated on Vero cells [21]. The skin sample from the male was highly positive with a $\mathrm{Ct}$ of 25 but no Besnoitia DNA was detected in skin sample from the female.

\section{Species identification using partial rDNA sequencing}

Following qPCR results, only the positive Besnoitia DNA sample was used for specific identification. A portion of ribosomal DNA cistron containing 5 genes [18S, ITS1, 5.8S, internal transcribed spacer 2 (ITS2) and 28S] was amplified using the primers Bes-F and Bes-R as designed by others [7] and synthesized by Eurogentec (Angers, France). The PCR reaction was performed in a total volume of $50 \mu \mathrm{l}$ per sample with $5 \mu$ DNA template, $10 \mathrm{pmol}$ of each primer and $25 \mu \mathrm{l}$ of the Taq PCR Master Mix ${ }^{\odot}$ (Qiagen) containing (final concentrations) $1.5 \mathrm{mM} \mathrm{MgCl}_{2}$, $200 \mu \mathrm{M}$ of each dNTP, 1.25 units of Taq polymerase and Qiagen PCR Buffer $1 \times(\mathrm{pH}=8.7)$. The PCR was performed in a GeneTouch Thermal Cycler (Bioer, Hangzhou, China) using the following program: $94{ }^{\circ} \mathrm{C}$ for 3 min (initial denaturation), 35 cycles at $94^{\circ} \mathrm{C}$ for $45 \mathrm{~s}$, annealing at $61{ }^{\circ} \mathrm{C}$ for $60 \mathrm{~s}$ and extension at $68^{\circ} \mathrm{C}$ for $60 \mathrm{~s}$, with a final extension step at $68{ }^{\circ} \mathrm{C}$ for $10 \mathrm{~min}$. Positive and negative controls were the same as those used for qPCR. PCR products were sequenced directly in both directions with the primers used for DNA amplification at the genomic facility GeT-Purpan (Federative Institute for Bio-medical research, Toulouse, France). The 938 bp rDNA sequence containing complete sequences of $5.8 \mathrm{~S}$ and internal transcribed spacers (ITS1 and ITS2), and partial sequences of $18 S$ and $28 S$ is deposited in GenBank under the accession number MG652473. This sequence was aligned to other sequences of besnoitiosis agents available in the GenBank database, B. besnoiti (DQ227419, DQ227418, AY833646, DQ227420) and B. caprae (HM008988), using Muscle in the Mega 7 software [22] (Fig. 5). Sequences of Toxoplasma gondii (GenBank: X75429) and Neospora caninum (GenBank: GQ899204) were also included as outgroup. Phylogenetic relationships

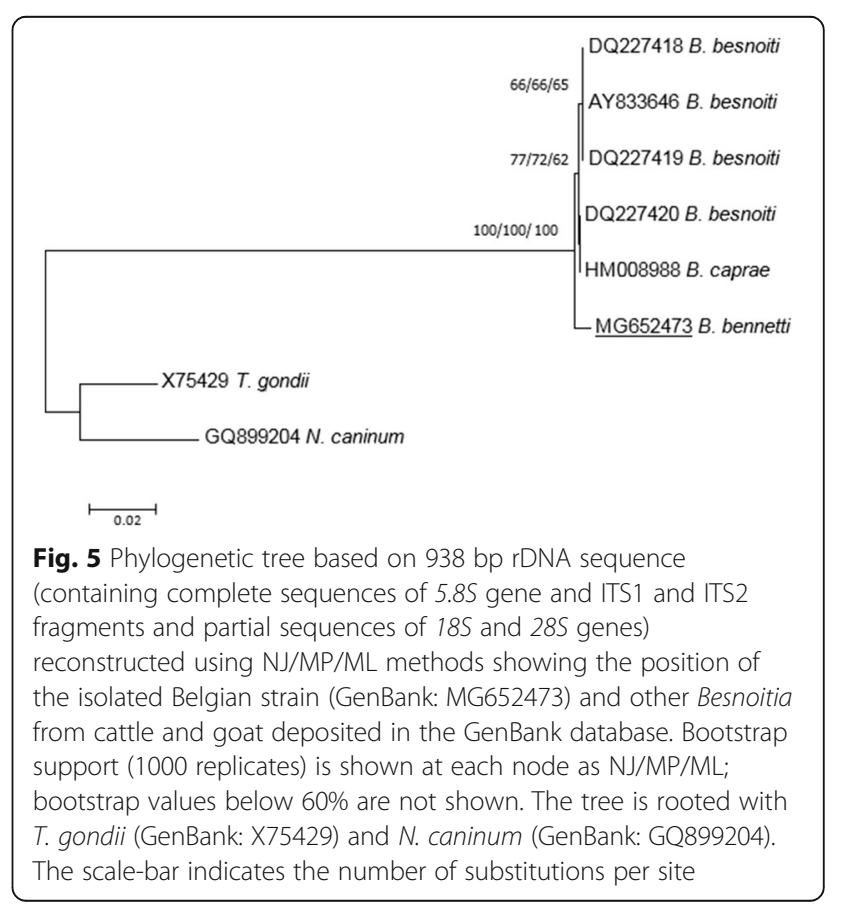


were inferred using three different molecular methods: neighbour joining (NJ), maximum parsimony (MP) and maximum likelihood (ML) with selection of the best model for nucleotide substitution by the Find Best DNA Model test implemented in Mega 7 (for NJ and ML methods). Reliability of the phylogenetic trees using Tamura-3 parameter + I model (NJ and ML) was tested using 1000 bootstrap replicates (NJ, ML and MP).

Besnoitia bennetti infection was confirmed by the ITS1 sequencing. The ITS1 sequence ( $244 \mathrm{bp})$ was completely identical with $B$. bennetti ITS1 sequences already deposited in the GenBank database (AY827839, AY665399 and JQ013812). ITS1 sequence comparisons between B. bennetti, B. besnoiti, B. caprae and B. tarandi have been previously performed [18] and emphasized the close relationship between besnoitiosis agents infecting ungulates [7]. Moreover, as $5.8 S$ (164 bp), ITS2 (393 $\mathrm{bp})$ and partial $18 S(56 \mathrm{bp})$ and $28 S(81 \mathrm{bp})$ sequences are not currently available in the GenBank database for $B$. bennetti, phylogenetic tree reconstructions were performed using this partial 938 bp rDNA sequence with $B$. besnoiti and B. caprae rDNA sequences. The phylogenetic tree reconstructed using the NJ method supported an identical topology to that of the ML and MP analyses (Fig. 5). These trees confirmed that B. bennetti isolated from the equid host (donkey) was a distinct species to Besnoitia species (B. besnoiti and B. caprae) from bovid hosts (cattle and goat, respectively).

Taking all these results a diagnosis of besnoitiosis was established in both animals.

\section{Control}

A daily treatment based on sulfamethoxazole and trimethoprim (Emdotrim 60\% Mix ${ }^{\oplus} 30 \mathrm{mg} / \mathrm{kg}$ ) was given orally to the affected animal and some improvement was noticed.

\section{Outcome}

Following the diagnosis, the affected animal was treated for four months with sulfamethoxazole and trimethoprim alongside a discontinued hepatoprotective treatment $\left(\right.$ Sédochol $\left.{ }^{\oplus}\right)$. The animal gained weight and no more skin conditions were visible.

\section{Discussion}

The current gold standard for diagnosing besnoitiosis in donkeys is histological identification of Besnoitia cysts within the dermis of individuals with lesions, generally achieved via skin biopsy [15, 23-25]. The size of the cyst and its wall were compatible with $B$. bennetti. Indeed, reported measurement are as follows: 150-450 $\mu \mathrm{m}$ in diameter and $20-50 \mu \mathrm{m}$ for the cyst wall $[18,23]$; however, others [26] reported a thinner cyst wall $(<7 \mu \mathrm{m})$. This morphological description is also compatible with
B. besnoiti in cattle with cysts up to $600 \mu \mathrm{m}$ in diameter $[2,27]$. The nares and the sclera are the most common locations for Besnoitia spp. cysts in donkeys [18]; the presence of cysts in the buccal mucosa has been rarely described [28]. Visualizing cysts in two (nares and scleral) or more locations correctly identified $83 \%$ of infected donkeys [16]. Serology is less invasive than histological examination and is a better choice for detection of subclinical infection [16]. However, some limitations have been pointed out when it is used alone. Some animals were not detected despite exhibiting severe clinical signs with numerous tissue cysts [16] and it was not possible to discriminate between $B$. besnoiti and B. bennetti infection by serology [29].

Ribosomal DNA ITS1 sequences are only available for $B$. bennetti isolated from donkeys in the USA. No ITS1 sequence variation was found between Belgian and USA samples with the same $\mathrm{T}$ insertion at position 148 (Additional file 1). The insertion was not present in any other Besnoitia species in ungulates (Additional file 1) leading to an identity of $99.7 \%$ with B. besnoiti, B. caprae and $B$. tarandi [18]. Microsatellites were also used to assess genetic variability between $B$. tarandi, B. besnoiti and $B$. bennetti involving intraspecific variations $[4,29,30]$. However, the two $B$. bennetti samples used for these analyses and provided by $[17,18]$ were previously identified by morphological features and ITS1 sequences [17, 18]. Moreover, in these two studies and for the present work, only one sample of $B$. bennetti was included, preventing investigation of intra-specific variations. To date, no ITS2, 5.8S, partial and conserved $18 S$ and $28 S$ sequences were available for $B$. bennetti. Consequently, this study gives new information concerning these domains, highlighting site variations between $B$. bennetti and Besnoitia species infecting other ungulates, leading to identification of a distinct genotype of $B$. bennetti. ITS2 sequence comparison with sequences of Besnoitia infecting cattle and goats showed only three different base pairs in the ITS2 domain, two different base pairs in the 5.85 domain (Additional files 2 and 3) and three to four randomly distributed base exchanges for $18 \mathrm{~S}$ and $28 S$ rDNA, respectively (data not shown). Despite the low level of nucleotide differences between Besnoitia agents infecting domestic ungulates, the phylogenetic tree reconstructions showed that $B$. bennetti was not included in the clade involving Besnoitia species from cattle and goat with strong supports. This separation was congruent with previous microsatellite studies $[4,29,30]$.

Moreover, adding biological features including host range as reviewed by [2] has allowed the collection of evidence to identify $B$. bennetti in this Belgian donkey. The whole genome sequencing of $B$. besnoiti will perhaps provide new molecular targets useful for species phylogeny and genetic population structure. 
Concerning haematological evaluation, anaemia was also described but without eosinophilia [23]. However, very little data exists in the literature and these parameters do not seem to be of valuable assistance for diagnosis. Nevertheless, it could be useful for a correct evaluation of renal function before sulfamethoxazole-trimethoprim treatment.

No efficient treatment for besnoitiosis is available. Clinical improvement observed here could be spontaneous and similar to apparent recovery observed in cattle without treatment [3].

In Europe, clinical cases have been reported in France in 1922 [31] and in Spain based on clinical signs and cyst morphology [25]. A very recent serological survey in Spain combining ELISA and western blot confirmation detected antibodies against Besnoitia spp. in equids (donkeys, mules and horses) [29]. Unfortunately, neither isolation of parasites nor further molecular genotyping were achieved to determine the accurate Besnoitia species involved in these three studies.

This first report of besnoitiosis in donkeys in Belgium suggests an urgent need for an extensive serological survey to assess prevalence in not only Belgian donkey populations but also in France, due to the geographical origin of the mare.

\section{Conclusions}

Difficulties in confirming diagnoses and the absence of efficient treatment options are probably responsible for the underreporting of besnoitiosis in European donkeys. This report aimed to highlight to clinicians the necessity to include besnoitiosis in the differential diagnosis of chronic dermatitis unresponsive to routine topical and systemic treatments. Whether this finding represents an unusual cluster of infections or reflects a wider distribution of subclinical infections which have largely gone undetected to date requires further study. Donkeys are increasing in number and particularly in developing countries [36]. Widespread distribution of this infection would be of international concern and veterinary medical importance. This case report is the first unequivocal report of $B$. bennetti infection of donkey in Europe based on clinical, histological, serological and molecular tools. Further investigations are necessary to unravel the phylogeny of B. bennetti and its epidemiology in Europe and elsewhere.

\section{Additional files}

Additional file 1: Alignment of ITS1. Variable sites are highlighted in yellow. (DOCX $116 \mathrm{~kb}$ )

Additional file 2: Alignment of ITS2. Variable sites are highlighted in yellow. (DOCX $112 \mathrm{~kb}$ )
Additional file 3: Alignment of 5.85. Variable sites are highlighted in yellow. (DOCX $66 \mathrm{~kb}$ )

\section{Abbreviations}

EFSA: European Food and Safety Authority; ELISA: Enzyme-linked immunosorbent assay; ITS: Internal transcribed spacer; ML: Maximum likelihood; MP: Maximum parsimony; NJ: Neighbour joining; PCR: Polymerase chain reaction; qPCR: Quantitative PCR; rDNA: Ribosomal DNA

\section{Acknowledgments}

We would like to thank Colette de Vincke, the owner of the two donkeys, for her kindness and help in handling the patients, and Amelia Coggon for review and improvement of the English language.

\section{Availability of data and materials}

The datasets used and/or analysed during the current study are available from the corresponding author upon reasonable request.

\section{Authors' contributions}

EL and YC wrote the manuscript. EL, EB, FP and SS performed serology and molecular biology. IT and CV performed the clinical part of the study. SV performed the histopathological part of the study. BL and MF read and improved the manuscript. YC supervised all the steps of the study. All authors read and approved the final manuscript.

Ethics approval and consent to participate Not applicable.

\section{Consent for publication}

Not applicable.

\section{Competing interests}

The authors declare that they have no competing interests.

\section{Publisher's Note}

Springer Nature remains neutral with regard to jurisdictional claims in published maps and institutional affiliations.

\section{Author details}

${ }^{1}$ Laboratoire de Parasitologie et Maladies Parasitaires, ENVT, Université de Toulouse, Toulouse, France. ${ }^{2}$ HHAP, INRA, ENVT, Université de Toulouse, Toulouse, France. ${ }^{3}$ Waterloo, Belgium. ${ }^{4}$ University of Ghent, Faculty of Veterinary Medicine, Merelbeke, Belgium. ${ }^{5}$ University of Liège, Faculty of Veterinary Medicine, Liège, Belgium.

Received: 7 February 2018 Accepted: 4 July 2018

Published online: 18 July 2018

\section{References}

1. European Food Safety Authority. Bovine besnoitiosis: an emerging disease in Europe. EFSA J. 2010:8:1499.

2. Olias P, Schade B, Mehlhorn H. Molecular pathology, taxonomy and epidemiology of Besnoitia species (Protozoa: Sarcocystidae). Infect Genet Evol. 2011;11:1564-76.

3. Alvarez-Garcia G, Frey CF, Mora LMO, Schares G. A century of bovine besnoitiosis: an unknown disease re-emerging in Europe. Trends Parasitol. 2013;29:407-15.

4. Gutiérrez-Expósito D, Arnal MC, Martínez-Durán D, Regidor-Cerrillo J, Revilla M, Fernández de Luco DL, et al. The role of wild ruminants as reservoirs of Besnoitia besnoiti infection in cattle. Vet Parasitol. 2016;223:7-13.

5. Schares G, Basso W, Majzoub M, Rostaher A, Scharr JC, Langenmayer MC, et al. Comparative evaluation of immunofluorescent antibody and new immunoblot tests for the specific detection of antibodies against Besnoitia besnoiti tachyzoites and bradyzoites in bovine sera. Vet Parasitol. 2010;171: $32-40$.

6. Cortes H, Leitao A, Gottstein B, Hemphill A. A review on bovine besnoitiosis: a disease with economic impact in herd health management, caused by Besnoitia besnoiti (Franco and Borges). Parasitology. 2014;141:1406-17.

7. Namazi F, Oryan A, Sharifiyazdi H. Genetic characterization of the causative agent of besnoitiosis in goats in Iran on the basis of internal transcribed 
spacer rDNA and its comparison with Besnoitia species of other hosts. Parasitol Res. 2011;108:633-8.

8. Vanhoudt A, Pardon B, De Schutter P, Bosseler L, Sarre C, Vercruysse J, et al. First confirmed case of bovine besnoitiosis in an imported bull in Belgium. Vlaams Diergeneeskd Tijdschr. 2015;84:205-11.

9. Bennett SC. A peculiar equine Sarcosporidium in the Anglo-Egyptian Sudan. Vet J. 1927;83:297-304.

10. Schultz KCA, Thorburn JA. Globidosis - a cause of dermatitis in horses. J South Afr Vet Med Assoc. 1955;26:39-43.

11. Bigalke RD. Studies on equine besnoitiosis. J Parasitol. 1970;56:29.

12. van Heerden J, Els HJ, Raubenheimer EJ, Williams JH. Besnoitiosis in a horse. J South Afr Vet Assoc. 1993;64:92-5.

13. Smith $H A$, Jones $T C$, Hunt RD. Diseases due to protozoa. Veterinary Pathology. 4th ed. Philadelphia: Lea and Febiger; 1972. p. 689-701.

14. Terrell TG, Stookey UL. Besnoitia bennetti in two Mexican burros. Vet Pathol. 1973;10:177-84.

15. Wendell PD, Duncan FP, Dunstan RW. Besnoitiosis in a miniature donkey. Vet Dermatol. 1997;8:139-43.

16. Ness SL, Schares G, Peters-Kennedy J, Mittel LD, Dubey JP, Bowman DD, et al. Serological diagnosis of Besnoitia bennetti infection in donkeys (Equus asinus). J Vet Diagn. 2014;26:778-82.

17. Elsheikha HM. Besnoitia bennetti infection in miniature donkeys: an emerging protozoan of increasing concern. Vet Parasitol. 2007;145:390-1.

18. Ness SL, Peters-Kennedy J, Schares G, Dubey JP, Mittel LD, Mohammed HO, et al. Investigation of an outbreak of besnoitiosis in donkeys in northeastern Pennsylvania. J Am Vet Med Assoc. 2012:240:1329-37.

19. Cortes HCE, Nunes $S$, Reis Y, Staubli D, Vidal R, Sager H, et al. Immunodiagnosis of Besnoitia besnoiti infection by ELISA and Western blot. Vet Parasitol. 2006;141:216-25.

20. Liénard E, Pop L, Prevot F, Grisez C, Mallet V, Raymond-Letron I, et al. Experimental infections of rabbits with proliferative and latent stages of Besnoitia besnoiti. Parasitol Res. 2015;114:3815-26.

21. Liénard E, Salem A, Jacquiet P, Grisez C, Prevot F, Blanchard B, et al. Development of a protocol testing the ability of Stomoxys calcitrans (Linnaeus, 1758) (Diptera: Muscidae) to transmit Besnoitia besnoiti (Henry, 1913) (Apicomplexa: Sarcocystidae). Parasitol Res. 2013;112:479-86.

22. Kumar S, Stecher G, Tamura K. MEGA7: Molecular Evolutionary Genetics Analysis version 7.0 for bigger datasets. Mol Biol Evol. 2016;33:1870-4.

23. Dubey JP, Sreekumar C, Donovan T, Rozmanec M, Rosenthal BM, Vianna MCB, et al. Redescription of Besnoitia bennetti (Protozoa: Apicomplexa) from the donkey (Equus asinus). Int J Parasitol. 2005;35:659-72.

24. Elsheikha HM, Mansfield LS, Morsy GH. Studies on besnoitiosis bennetti in miniature donkeys. J Egypt Soc Parasitol. 2008;38:171-84.

25. Zafra R, Soria-Lopez N, de Castro ED, Jaber J, Mozos E, Pérez J. Outbreak of besnoitiosis in donkeys (Equus asinus) in the south of Spain. J Comp Pathol. 2013;1:81.

26. Elsheikha HM, Murphy AJ, Mansfield LS. Phylogenetic congruence of Sarcocystis neurona Dubey et al., 1991 (Apicomplexa: Sarcocystidae) in the United States based on sequence analysis and restriction fragment length polymorphism (RFLP). Syst Parasitol. 2005;61:191-202.

27. Langenmayer MC, Gollnick NS, Majzoub-Altweck M, Scharr JC, Schares G, Hermanns W. Naturally acquired bovine besnoitiosis: histological and immunohistochemical findings in acute, subacute, and chronic disease. Vet Pathol. 2015;52:476-88.

28. Elsheikha HM, Mackenzie CD, Rosenthal BM, Marteniuk JV, Steficek B, Windsor $\mathrm{S}$, et al. An outbreak of besnoitiosis in miniature donkeys. J Parasitol. 2005;91:877-81.

29. Gutiérrez-Expósito D, García-Bocanegra I, Howe DK, Arenas-Montes A, Yeargan MR, Ness SL, et al. A serosurvey of selected cystogenic coccidia in Spanish equids: first detection of anti-Besnoitia spp. specific antibodies in Europe. BMC Vet Res. 2017;13:128

30. Madubata C, Dunams-Morel DB, Elkin B, Oksanen A, Rosenthal BM. Evidence for a recent population bottleneck in an apicomplexan parasite of caribou and reindeer, Besnoitia tarandi. Infect Genet Evol. 2012;12:1605-13.

31. Henry A, Masson G. Traité de protozoologie médicale et vétérinaire. Paris: Vigot frères; 1922

\section{Ready to submit your research? Choose BMC and benefit from:}

- fast, convenient online submission

- thorough peer review by experienced researchers in your field

- rapid publication on acceptance

- support for research data, including large and complex data types

- gold Open Access which fosters wider collaboration and increased citations

- maximum visibility for your research: over $100 \mathrm{M}$ website views per year

At BMC, research is always in progress.

Learn more biomedcentral.com/submissions 